\title{
Treatment of posttraumatic lumbar interspinous ligament calcification with partial resection of spinous processes and calcified interspinous ligaments: case report
}

\author{
Angela Mae Richardson, MD, PhD, Joanna Elizabeth Gernsback, MD, John Paul G. Kolcun, BS, \\ and Steven Vanni, DO, DC
}

Department of Neurosurgery, University of Miami, Jackson Memorial Hospital, Miami, Florida

The authors report on the first surgical treatment for traumatic interspinous ligament calcification, with significant radiographic and symptomatic improvements at long-term follow-up. Heterotopic ossification occurs following traumatic injury but does not typically affect the interspinous ligaments. While these ligaments can calcify with age, this is rarely seen in patients younger than 50 years of age. The authors present the unusual case of a 31-year-old man who suffered traumatic fractures of thoracic and lumbar spinous processes. He developed progressive low-back pain that failed to respond to conservative treatments. At presentation, he was neurologically intact. CT scanning demonstrated partial calcification of the interspinous ligaments at L2-3, L3-4, and L4-5 with significant hypertrophy of the spinous processes at those levels. He did not have significant disc pathology, and his symptoms were attributed to the limited range of motion caused by the enlarged spinous processes. Partial resection of the spinous processes and calcified interspinous ligaments was performed to remove the heterotopic bone. The patient was seen in follow-up at 5 months postoperatively for imaging, and he was interviewed at 1 and 2 years postoperatively. He is doing well with significant pain relief and an improved range of motion. His Oswestry Disability Index improved from 25 preoperatively to 18 at 2 years postoperatively.

https://thejns.org/doi/abs/10.3171/2018.9.SPINE18401

KEYWORDS heterotopic bone; interspinous ligament calcification; posttraumatic; treatment; lumbar

$\mathrm{T}$ HE interspinous ligament spans adjacent spinous processes in a posterocranial direction. This ligament is composed of ventral, middle, and dorsal portions. The ventral part is an extension of the ligamentum flavum, reaching posteriorly and cranially to attach to the undersurface of the spinous process above. ${ }^{2}$

Calcification of the interspinous ligaments increases with age; a cohort study identified calcification on CT at this site in $0 \%$ of patients younger than age 50 years, $5 \%$ of those 50-60 years, $7.3 \%$ of those $60-70$ years old, and $11 \%$ of those over the age of $70 .{ }^{1}$ Although the study did not identify calcifications in any patients under 50 years of age, ossification in this population has been occasionally reported in the literature. Patients with severe vitamin Dresistant rickets, patients with prior surgery involving the posterior spinous ligaments, and one young athlete with spontaneous calcification have all been reported. .,7,9 $^{2}$

Heterotopic ossification, the formation of extraskeletal lamellar bone, occurs after traumatic injuries and burns, as the inflammatory cascade initiated by these insults also activates pro-osteogenic factors. ${ }^{4}$

Here we present the first reported case of trauma-induced calcification of the interspinous ligament in a young man with progressive back pain. His symptoms and range of motion were significantly improved following operative resection of the heterotopic bone.

\section{Case Report}

History

A 31-year old man initially presented to an outside hospital following a motorcycle crash in 2009. At the time, he complained of low-back pain with radiation to the leg, but he was neurologically intact. He underwent abdominal $\mathrm{CT}$ as part of the trauma protocol. This scan demonstrated normal morphology of his vertebral bodies, but with acute fractures of the spinous processes at L1-5 (Fig. 1). MRI revealed a small disc bulge at L5-S1 without significant 


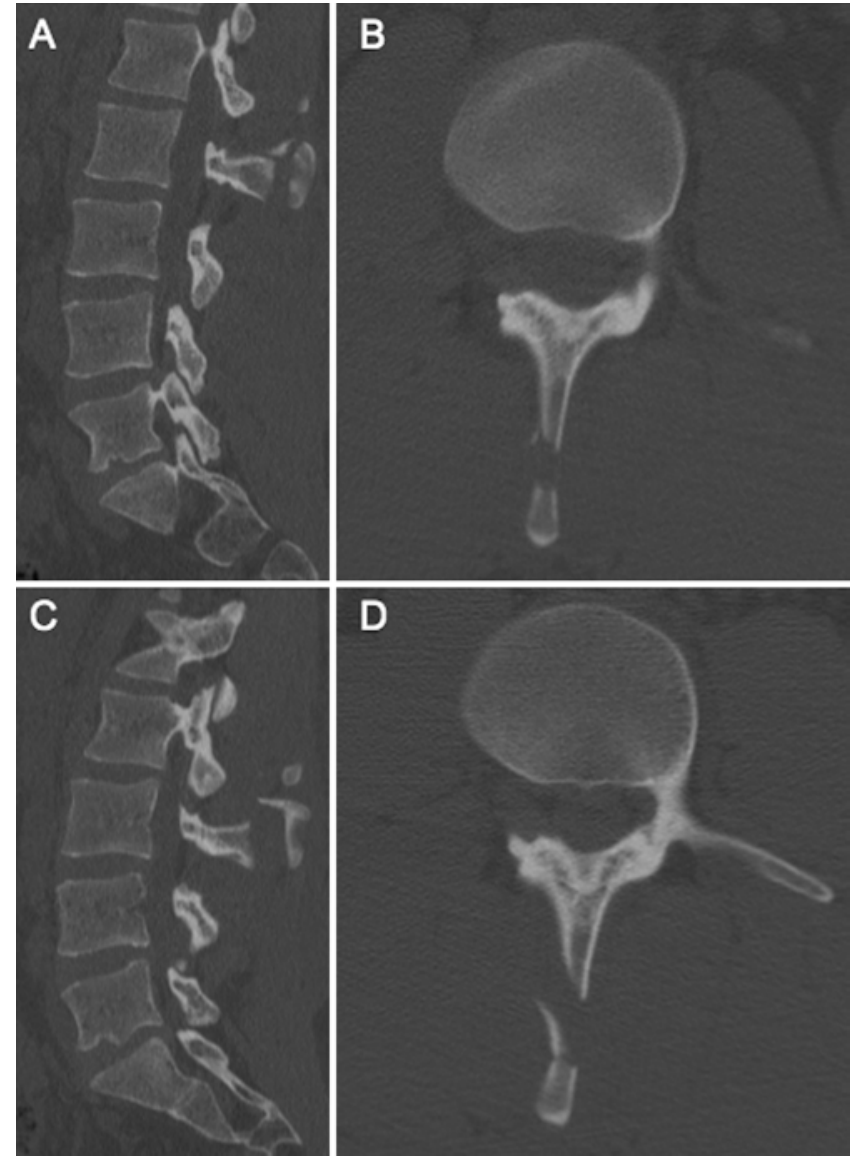

FIG. 1. Abdominal CT obtained at presentation. A: Sagittal view demonstrating an L2 spinous process fracture. B: Axial view through the fractured $\mathrm{L} 2$ spinous process. C: Sagittal view demonstrating an $\mathrm{L} 3$ spinous process fracture. D: Axial view through the fractured $L 3$ spinous process.

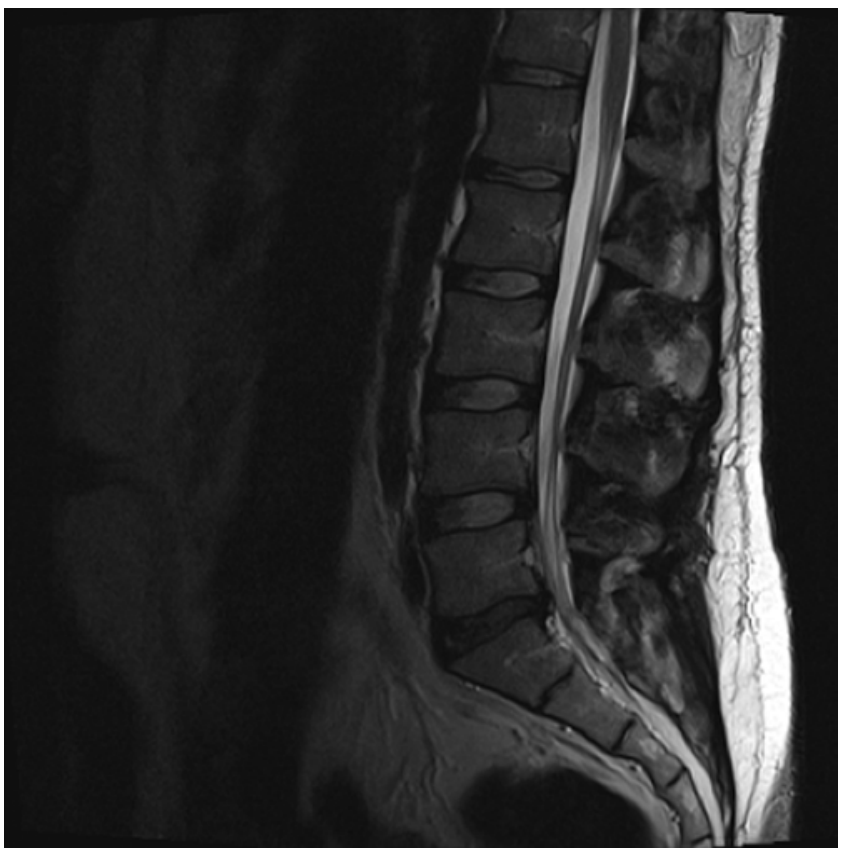

FIG. 2. Sagittal T2-weighted MR image of the lumbar spine demonstrating a patent spinal canal with a black disc at L5-S1 with a mild bulge posteriorly. No significant compression of the thecal sac is visible.
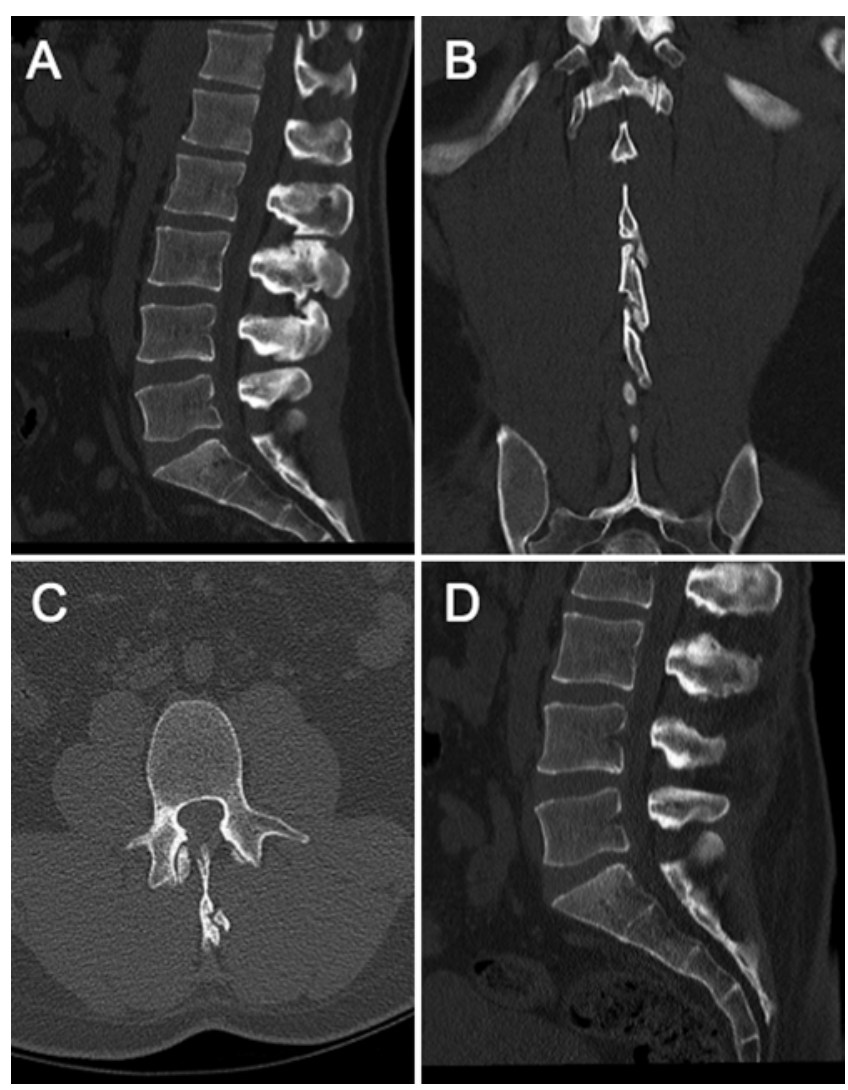

FIG. 3. Preoperative sagittal (A), coronal (B), and axial (C) lumbar spine CT scans without contrast showing calcification of the interspinous and paraspinal ligaments with close apposition of adjacent spinous processes. Representative sagittal image (D) obtained at 2 years postoperatively, demonstrating the extent of resection of hypertrophic spinous processes and surrounding tissues.

compression of the thecal sac or nerve root. His pain was managed conservatively with improvement over time. He experienced an exacerbation of his chronic back pain following another motor vehicle accident in 2015. Imaging at that time did not identify any acute pathology but did demonstrate enlargement of the spinous processes and calcification of the interspinous ligament.

\section{Examination}

He returned to our clinic several months later with persistent progressive severe back pain. He had aching in his lower back and stabbing pain at the lumbosacral junction. He remained neurologically intact but had a decreased range of motion, particularly in extension. The previously identified disc bulge was stable on repeat MRI (Fig. 2). We recommended CT of the lumbar spine and flexion-extension lumbar radiographs. The CT scan demonstrated calcification of the interspinous ligaments from L2 to L5 with heterotopic ossification in the paraspinal ligaments and hypertrophy of the spinous processes (Fig. 3A-C). He had significantly limited range of motion on flexion-extension radiographs (Fig. 4A-C).

\section{Operation}

As conservative management had failed, we considered 


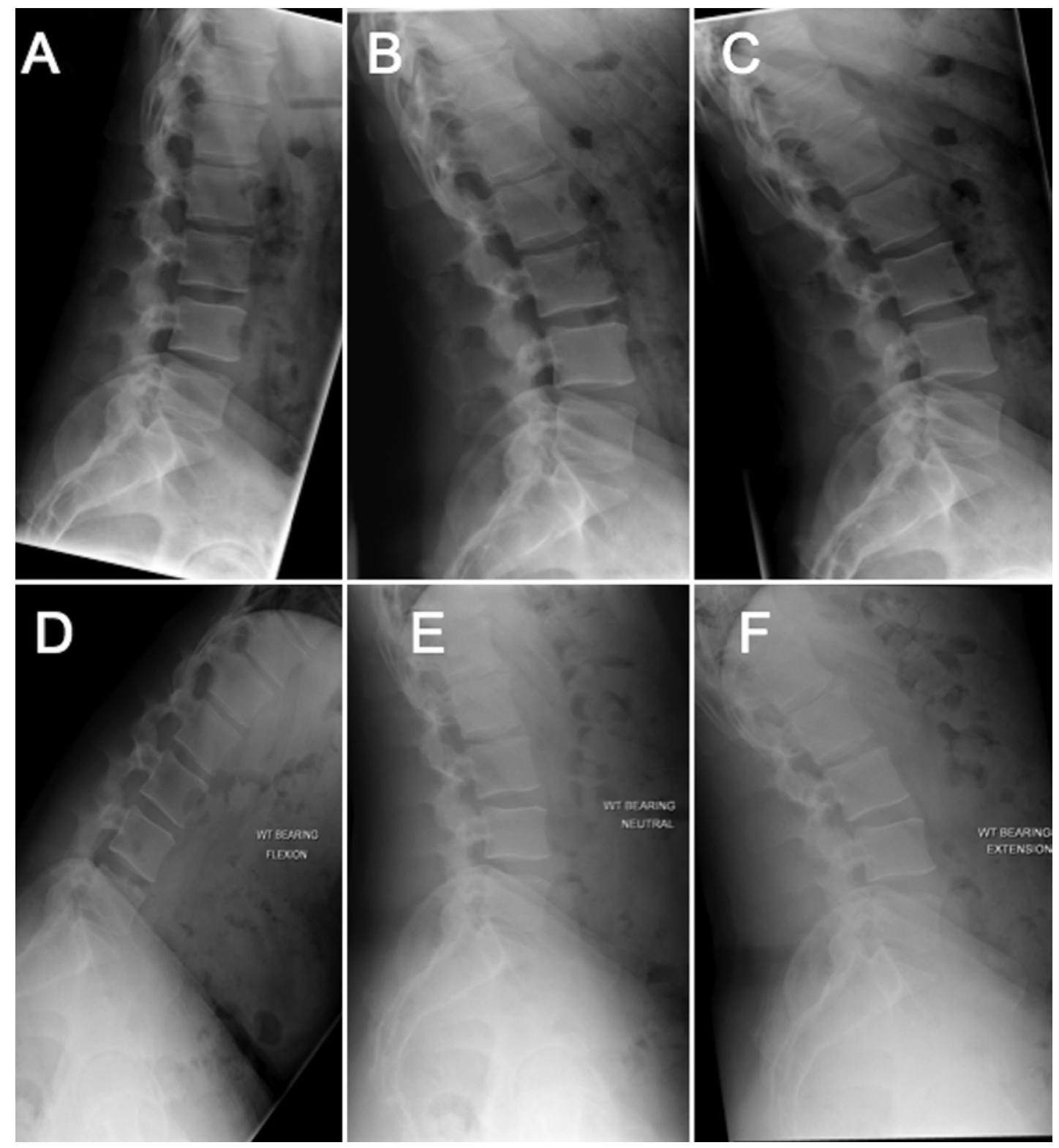

FIG. 4. Preoperative lateral radiographs of the lumbar spine obtained on flexion (A), in the neutral position (B), and on extension (C), showing a limited range of motion on extension. Five-month postoperative radiographs obtained on flexion (D), in the neutral position $(\mathbf{E})$, and on extension $(\mathbf{F})$, showing a notably increased range of motion on extension.

surgical options for his case. Initially, when only his MRI study was available, we discussed fusing L5-S1 with either a transforaminal lumbar interbody fusion (TLIF) or anterior lumbar interbody fusion (ALIF) in order to treat the degenerated disc. He returned to clinic with a CT demonstrating heterotopic ossification throughout his lumbar spine. This finding in combination with radiographs showing a limited range of motion led us to hypothesize that the pain generator was limited motion in extension due to the excessive bone formation of the spinous processes with calcification of the surrounding ligaments.

To remove the abnormal bone preventing his ability to extend his spine, we performed a partial resection of the spinous processes at L2-3, L3-4, and L4-5, with removal of the heterotopically calcified interspinous ligament in its entirety along with a small portion of the surrounding spinous processes. Pathology demonstrated normal bone with unremarkable marrow.

\section{Postoperative Course}

He was discharged with a prescription for ibuprofen to prevent additional heterotopic ossification and fusion. He was seen in clinic at 2 weeks, 6 weeks, and 5 months postoperatively. He reported improvement in back pain at all visits and demonstrated an increased range of motion on lumbar radiographs without the formation of new bone (Fig. 4D-F). His preoperative lumbar lordosis was $38^{\circ}$ with $6^{\circ}$ of motion between neutral and extension. At 5 months after surgery, his lumbar lordosis was $44^{\circ}$ with $28^{\circ}$ 


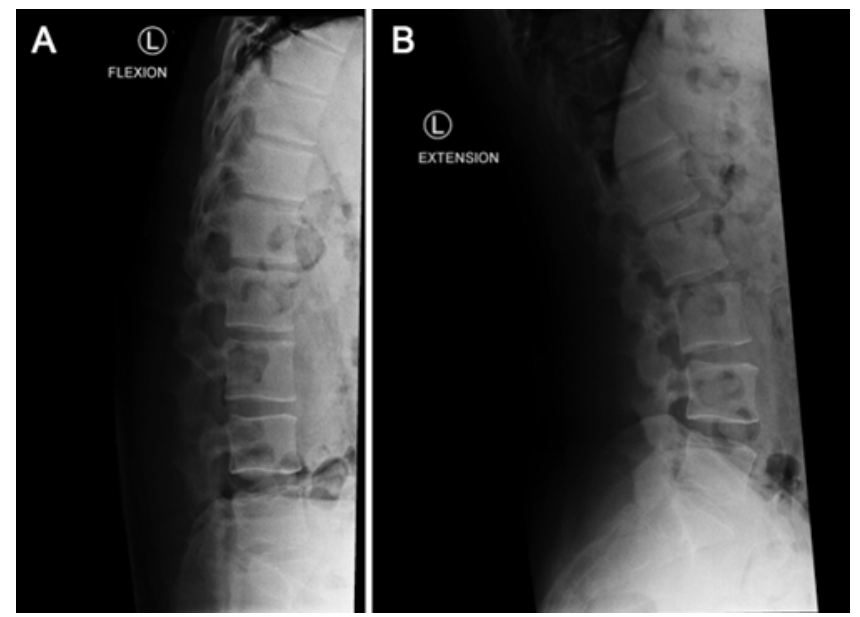

FIG. 5. Two-year postoperative lateral radiographs of the lumbar spine obtained on flexion (A) and extension (B), showing preserved range of motion.

of motion between neutral and extension. His preoperative Oswestry Disability Index (ODI) had been 25. On interview at 3,12 , and 24 months after surgery, his ODI had improved to 16,15 , and 18, respectively. At 2 years after surgery, he reported no symptoms aside from soreness in the lower back after prolonged strenuous physical activity. The soreness resolves over the subsequent day and is adequately controlled with ibuprofen. He is able to exercise regularly without discomfort. We believe that the reduction in his back pain is attributable to his improved range of motion from the removal of the heterotopic bone. Repeat imaging at 2 years postoperatively demonstrated the absence of hypertrophic calcified ligament (Fig. 3D) and preserved range of motion in flexion and extension (Fig. 5).

\section{Discussion}

This case demonstrates a rare development of paraspinal heterotopic ossification with calcification of the interspinous ligament following blunt trauma. The patient was treated successfully with a conservative surgery, with significant symptom relief at the long-term follow-up.

Heterotopic ossification is commonly seen in the long bones, hips, and shoulders following traumatic injuries and burns and occurs in 10\%-53\% of patients after traumatic brain or spinal cord injury. ${ }^{6}$ An isolated case of heterotopic ossification in the paraspinal muscles was reported in a soldier 3 years after a 30-ft fall. He initially developed a lumbar soft-tissue hematoma, without bony injury, that was managed conservatively. He developed progressive back pain in the years following his injury, and radiographs demonstrated paraspinal heterotopic ossification. ${ }^{3}$ In our patient, we saw ossification of the interspinous ligaments, which is commonly seen in the elderly but not in those under age 50..$^{1}$ The interspinous ligament contains nociceptors, and the injection of hypertonic saline into this structure produces significant pain, so the presence of abnormal calcification here, along with the patient's decreased range of motion, could be responsible for his pain. ${ }^{8}$

Interspinous ligament calcification of several etiologies has been reported, including spontaneous, postoper- ative, and genetic, but it has not been reported following trauma. Calcification can be seen in many spinal ligaments in patients with vitamin D-resistant rickets. However, the interspinous and supraspinous ligaments ossify only in a subset of those patients with mutations in the phosphateregulating neutral endopeptidase, $\mathrm{x}$-linked gene $(P H E X)$ who experience more severe disease overall. ${ }^{5}$ Postoperative calcification of the interspinous ligaments has been observed in a delayed fashion. Thirteen years after a bilateral laminotomy, a 51-year-old man presented with symptoms of cauda equina syndrome. Lumbar radiographs demonstrated a calcified mass in the area of prior surgery. He underwent CT myelography, which demonstrated compression of the thecal sac, and he was taken to surgery for decompression. His symptoms resolved within 3 weeks of surgery. ${ }^{9}$ Spontaneous calcification of interspinous ligaments has also been reported. A 21-year-old female collegiate gymnast presented with progressive back pain and an odd "gritty" sensation in her back. She was neurologically intact and had full range of motion. Ultrasound demonstrated a hyperechoic area consistent with calcification in the interspinous ligament between T12 and L1. The presumed calcification was fenestrated multiple times under ultrasound guidance, and a combination of lidocaine and triamcinolone was injected into the area. She noted immediate improvement in her back pain; the relief persisted at follow-up, and ultrasound demonstrated regression of the small area of calcification. ${ }^{7}$ In our patient, this procedure likely would not have been effective, as his disease was more extensive and the heavily mineralized ligaments would have made needle fenestration technically difficult. Resection of the calcified ligament led to improvement in both his pain and his range of motion.

Patients may develop heterotopic ossification following traumatic injuries. As the formation of bone is a dynamic process, the presentation may be that of delayed or progressive pain. CT scans and radiographs are essential adjuncts for diagnosis and for planning an intervention. MRI is useful for assessing the soft tissues and neural elements but may not adequately demonstrate the degree of abnormal ossification or if heterotopic ossification has occurred. In patients with progressive symptoms, resection of abnormal calcifications may provide symptom relief and improve range of motion.

\section{References}

1. Hameed F, Hunter DJ, Rainville J, Li L, Suri P: Prevalence of anatomic impediments to interlaminar lumbar epidural steroid injection. Arch Phys Med Rehabil 93:339-343, 2012

2. Heylings DJ: Supraspinous and interspinous ligaments of the human lumbar spine. J Anat 125:127-131, 1978

3. Kang DG, Potter BK, Lehman RA Jr: Lumbar paraspinal heterotopic ossification. Spine J 12:631-633, 2012

4. Kraft CT, Agarwal S, Ranganathan K, Wong VW, Loder S, Li J, et al: Trauma-induced heterotopic bone formation and the role of the immune system: A review. J Trauma Acute Care Surg 80:156-165, 2016

5. Lee SH, Agashe MV, Suh SW, Yoon YC, Song SH, Yang $\mathrm{JH}$, et al: Paravertebral ligament ossification in vitamin Dresistant rickets: incidence, clinical significance, and genetic evaluation. Spine (Phila Pa 1976) 37:E792-E796, 2012 
6. Ranganathan K, Loder S, Agarwal S, Wong VW, Forsberg J, Davis TA, et al: Heterotopic ossification: basic-science principles and clinical correlates. J Bone Joint Surg Am 97:1101-1111, 2015

7. Simpson S, Krabak BJ: Interspinous ligament calcification in a collegiate gymnast. PM R 6:742-745, 2014

8. Tsao H, Tucker KJ, Coppieters MW, Hodges PW: Experimentally induced low back pain from hypertonic saline injections into lumbar interspinous ligament and erector spinae muscle. Pain 150:167-172, 2010

9. Weiss MH, Spencer GE: Ossification of a lumbar interspinous ligament with compression of the cauda equina. A case report. J Bone Joint Surg Am 52:165-167, 1970

\section{Disclosures}

Dr. Vanni serves as a consultant to NuVasive Inc., Globus Medical Inc., and Spine Wave Inc.

\section{Author Contributions}

Conception and design: Richardson, Vanni. Acquisition of data: Gernsback, Kolcun, Vanni. Analysis and interpretation of data: Richardson, Gernsback, Vanni. Drafting the article: Richardson, Gernsback. Critically revising the article: Gernsback, Kolcun, Vanni. Reviewed submitted version of manuscript: Richardson, Kolcun, Vanni. Study supervision: Vanni.

\section{Correspondence}

Angela M. Richardson: University of Miami, Jackson Memorial Hospital, Miami, FL. angela.richardson@jhsmiami.org. 